\title{
Portable Prehospital Methods to Treat Near-Hypothermic Shivering Cold Casualties
}

\author{
Samuel J. Oliver, PhD; Jennifer L. Brierley, PhD; Philippa C. Raymond-Barker, MSc; Alberto Dolci, MSc; \\ Neil P. Walsh, PhD \\ From the Extremes Research Group, School of Sport, Health and Exercise Sciences, Bangor University, Bangor, United Kingdom.
}

\begin{abstract}
Objective.-To compare the effectiveness of a single-layered polyethylene survival bag $(\mathrm{P})$, a singlelayered polyethylene survival bag with a hot drink $(\mathrm{P}+\mathrm{HD})$, a multi-layered metalized plastic sheeting survival bag (MPS: Blizzard Survival), and a multi-layered MPS survival bag with 4 large chemical heat pads (MPS+HP: Blizzard Heat) to treat cold casualties.

Methods.-Portable cold casualty treatment methods were compared by examining core and skin temperature, metabolic heat production, and thermal comfort during a 3-hour, $0^{\circ} \mathrm{C}$ cold air exposure in 7 shivering, near-hypothermic men $\left(35.4^{\circ} \mathrm{C}\right)$. The hot drink $\left(70^{\circ} \mathrm{C}, \sim 400 \mathrm{ml}, \sim 28 \mathrm{~kJ}\right)$ was consumed at 0,1 , and 2 hours during the cold air exposure.

Results. - During the cold air exposure, core rewarming and thermal comfort were similar on all trials $(P=.45$ and $P=.36$, respectively). However, skin temperature was higher $(10 \%-13 \% ; P<$ .001 ; large effect sizes $d>2.7)$ and metabolic heat production lower $(15 \%-39 \% ; P<.05$; large effect sizes $d>9$ ) on MPS and MPS+HP than P and P+HD. The addition of heat pads further lowered metabolic heat production by $15 \%$ (MPS+HP vs MPS; $P=.05$; large effect size $d=.9$ ). The addition of the hot drink to polyethylene survival bag did not increase skin temperature or lower metabolic heat production.
\end{abstract}

Conclusions.- -Near-hypothermic cold casualties are rewarmed with less peripheral cold stress and shivering thermogenesis using a multi-layered MPS survival bag compared with a polyethylene survival bag. Prehospital rewarming is further aided by large chemical heat pads but not by hot drinks.

Key words: rewarming, hypothermia, thermogenesis, multiple trauma, frostbite, wilderness medicine

\section{Introduction}

To prevent hypothermia-related mortality, it is vital to develop portable methods to attenuate heat loss that casualties and first responders can use as part of prehospital care while they await more sophisticated medical facilities. ${ }^{1,2}$ Multiple layering that includes a vapor-proof barrier and insulation has recently been highlighted as important for cold protection. ${ }^{3,4}$ Unfortunately, being cumbersome and heavy $(2.5 \mathrm{~kg})$, the

This research was previously presented in part as an abstract at the American College of Sports Medicine annual meeting on June 2, 2012 in San Francisco, CA.

The opinions and assertions herein are the private views of the authors and do not construe official views of Bangor University or Blizzard Protection Systems Ltd.

Corresponding author: Samuel J. Oliver, PhD, Extremes Research Group, School of Sport, Health and Exercise Sciences, Bangor University, Bangor, LL57 2PZ, United Kingdom (e-mail: s.j.oliver@ bangor.ac.uk). methods used in these studies have limited portability. A more portable cold protection method option is a survival bag constructed of multiple layers of metalized plastic sheeting (MPS: Blizzard Survival weight $0.4 \mathrm{~kg}$ ). The effectiveness of this survival bag to protect shivering human cold casualties compared with other vapor-proof barriers remains unknown. Portable heat sources that may benefit cold casualties are limited to chemical heat pads and the ingestion of hot drinks, as body-to-body contact is ineffective in rewarming shivering cold casualties. ${ }^{5}$ Although a recommended practice to support shivering, ${ }^{1}$ no study has investigated whether hot drink ingestion has thermally beneficial effects for cold casualties. As cold drinks reduce core temperature and subjective thermal discomfort during exerciseinduced hyperthermia, it might be hypothesized that hot drinks may increase core temperature and thermal comfort in shivering cold casualties. ${ }^{6}$ Large chemical heat pads are more effective than spontaneous 
thermogenesis to rewarm persons with inhibited shivering ${ }^{7}$; however, it remains unclear if they are effective in rewarming shivering cold casualties. This study's objective was therefore to compare the effectiveness to treat cold casualties of a single-layered polyethylene survival bag, a single-layered polyethylene survival bag with a hot drink, a multi-layered metalized plastic sheeting survival bag, and a multi-layered MPS survival bag with 4 large chemical heat pads.

\section{Methods}

\section{STUDY DESIGN}

A crossover study was performed where participants completed all trials in a random order. Randomization was completed by SJO (www.randomizer.org). The study received University Ethics Committee approval.

\section{Participants}

Seven healthy men (mean \pm SD: age $21 \pm 3$ years; height $178 \pm 5 \mathrm{~cm}$; nude body mass $70.5 \pm 5.2 \mathrm{~kg}$; body fat $10 \pm 3 \%$ ) volunteered for the study after giving written informed consent. Participants reported no infection and did not take medication or nutritional supplements 6 weeks before or during the study. To standardize nutritional status and physical activity, participants completed a food and activity diary for the 24 hours before the first trial, which they then repeated before each subsequent trial. In the 24 hours before each trial, participants also consumed water equal to $35 \mathrm{ml}$ per kilogram body mass and refrained from alcohol and exhaustive exercise.

\section{EXPERIMENTAL PROCEDURES}

In 4 trials, participants were made near-hypothermic by cold water immersion, after which they completed a 3hour $0^{\circ} \mathrm{C}$ cold air exposure in an environmental chamber using 1 of 4 cold casualty treatments: a single-layered polyethylene survival bag (P: 3-mil $[\sim 0.08 \mathrm{~mm}$ thick] polyethylene, weight $0.25 \mathrm{~kg}$, packed size $24 \times 15 \times 1$ $\mathrm{cm}$; Figure 1A), a single-layered polyethylene survival bag with a hot drink (P+HD), a multi-layered MPS survival bag (MPS: Blizzard Survival bag, weight 0.39 $\mathrm{kg}$, packed size $21 \times 11 \times 4 \mathrm{~cm}$; Figure $1 \mathrm{~B}$ ), and a multi-layered MPS survival bag with 4 large chemical heat pads (MPS+HP: Blizzard Survival Heat bag, weight $1.90 \mathrm{~kg}$, packed size $30 \times 25 \times 7 \mathrm{~cm}$; surface area of each large chemical heat pad $24 \times 30 \mathrm{~cm}$ ). The fifth trial was a control (CON) where participants remained seated in an ambient temperature $20^{\circ} \mathrm{C}$ (relative humidity $[\mathrm{RH}] 41 \%$ ).
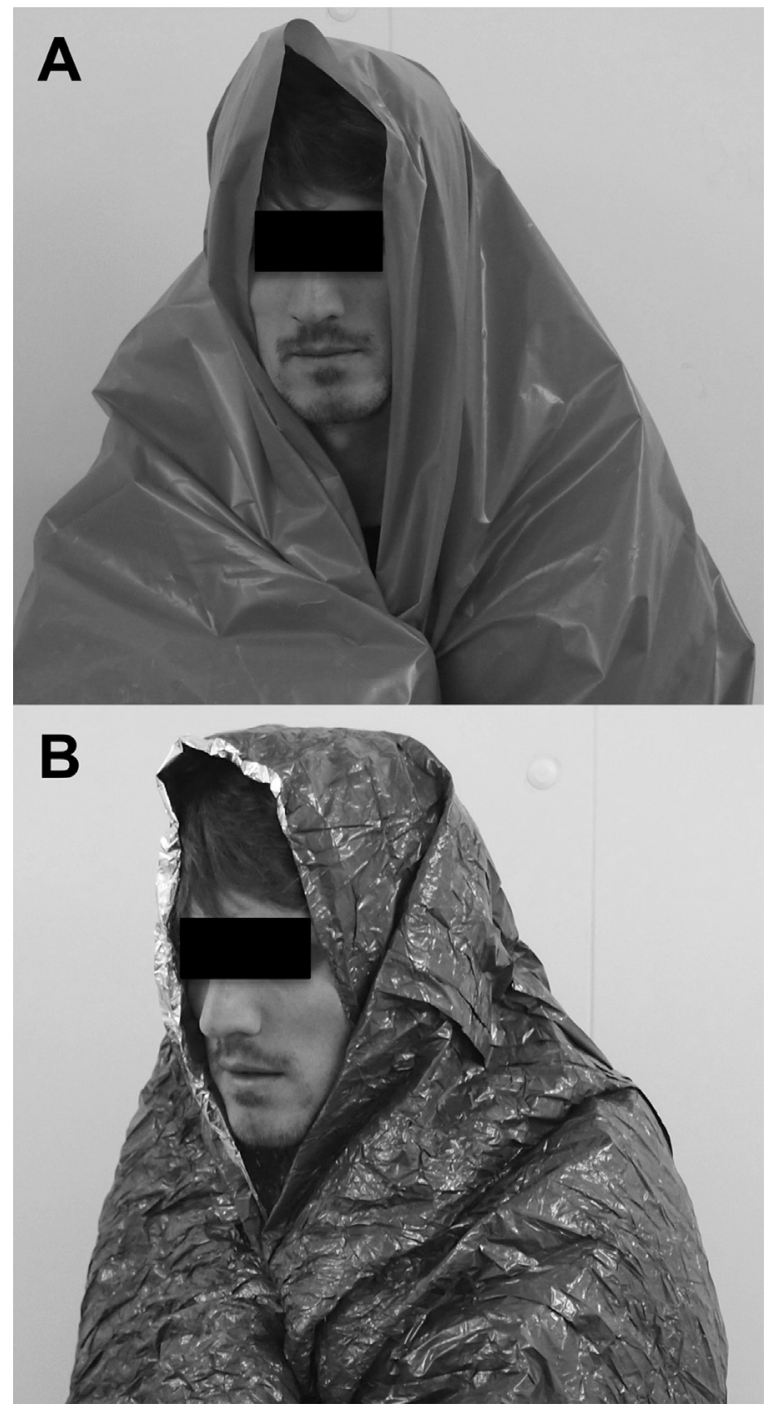

Figure 1. Polyethylene survival bag (A) and a multi-layered metallized plastic sheeting survival bag (B: Blizzard Survival bag).

After an overnight fast, participants arrived at 0800 hours. After voiding, anthropometric and body composition measures of height, body mass, and body fat were obtained (InBody230; Biospace, Seoul, South Korea). Urine was analyzed for specific gravity (Atago, Tokyo, Japan). Participants then fitted a rectal thermistor $12 \mathrm{~cm}$ beyond the anal sphincter (2020 Series; Grant Instruments, Cambridgeshire, UK) and began a 30-minute seated rest dressed in a tracksuit, swim shorts, t-shirt, socks, and shoes. On the cold stress trials, participants were then immersed up to the axilla in $13.0 \pm 0.1^{\circ} \mathrm{C}$ stirred water wearing swim shorts until core temperature reached $36^{\circ} \mathrm{C}$. After, participants were carefully dried, dressed in dry shorts, socks, and gloves, and fitted with skin thermistors. Participants then entered an environmental chamber and after 5 minutes seated were given, 
and instructed how to use, 1 of the portable cold casualty field methods. Participants remained seated for the 3 hours in the environmental chamber $\left(0^{\circ} \mathrm{C}, \mathrm{RH} 40 \%\right.$, and wind velocity $0.2 \mathrm{~m} / \mathrm{s}$; Delta Environmental Systems, West Yorkshire, UK). On CON, participants were fitted with rectal and skin thermistors and sat for the same duration as in the cold air exposure trials but were free to choose their attire. During all trials participants consumed flavored water from an insulated container equal to $6 \mathrm{~mL}$ per $\mathrm{kg}$ of body mass at 0,60 , and 120 minutes. The flavored water contained negligible energy content $(\sim 7 \mathrm{~kJ}$ per $100 \mathrm{~mL})$. Pilot testing confirmed that the $15-$ minute drinking period meant the average drink temperature consumed was $70^{\circ} \mathrm{C}$ on $\mathrm{P}+\mathrm{HD}$ and $36^{\circ} \mathrm{C}$ on all other trials. During the trials, core and skin temperature was measured continuously via thermometry. Mean skin temperature was calculated by an equation adjusted for regional proportions. ${ }^{8}$ Metabolic heat production and energy expenditure were determined by indirect calorimetry techniques and equations ${ }^{9,10}$ as described in detail previously. ${ }^{11}$ Thermal discomfort was determined by perceived thermal comfort (McGinnis 13-point scale: $1=$ so cold $\mathrm{I}$ am helpless, $7=$ comfortable, $13=$ so hot $\mathrm{I}$ am sick and nauseated), ${ }^{12}$ and pain sensation to cold (10-point scale: $1=$ barely cool to $10=$ unbearable pain). ${ }^{13}$ After cold air exposure, participants were rewarmed in $40^{\circ} \mathrm{C}$ water and monitored for 2 hours.

\section{STATISTICAL ANALYSES}

Based on the typical standard deviation of $0.6^{\circ} \mathrm{C}$ and $9 \mathrm{~W} / \mathrm{m}^{2}$ (co-efficient of variation $\sim 3 \%$ and $7 \%$, respectively), ${ }^{11}$ and using standard alpha (.05) and beta values (.8), a sample size of 7 was estimated (G*Power, Version 3.1.2) to have adequate statistical precision to detect a $0.8^{\circ} \mathrm{C}$ and $12 \mathrm{~W} / \mathrm{m}^{2}$ difference in skin temperature and metabolic heat production between any 2 conditions during the cold air exposure (2-tailed). The main outcome measures, skin temperature and metabolic heat production, were averaged using the arithmetic mean for each hour and compared between trials by 2 way fully repeated-measures analyses of variance (ANOVA). Significant main effects and interactions were explored by 1-way repeated measures ANOVA, uncorrected Fisher's LSD, and Tukey's post hoc tests. The meaningfulness of the differences between cold casualty treatment methods were quantified by Cohen's $d$ effect sizes, which can be interpreted as small (0.2), medium (0.5), and large (0.8). ${ }^{14}$ A 1-way repeated measures ANOVA was also used to compare baseline, cold water immersion time, and physiological responses at the start of the cold air exposure. Thermal discomfort was analyzed by Friedman test. Statistical significance was accepted at $P<.05$. All statistical analyses were completed using a computerized statistical software package (GraphPad Prism version 6; GraphPad Software, La Jolla, CA).

\section{Results}

\section{BASELINE PHYSIOLOGICAL RESPONSES AND COLD WATER IMMERSION TIMES}

At the start of the trials, body mass, body fat, urinespecific gravity, resting core temperature, and metabolic heat production were similar $(70.5 \pm 5.2 \mathrm{~kg}, P=.41 ; 10$ $\pm 3 \%, P=.55 ; 1.023 \pm 1.004, P=.69 ; 36.7 \pm 0.2^{\circ} \mathrm{C}$, $P=.73 ; 52 \pm 14 \mathrm{~W} / \mathrm{m}^{2}, P=.88$ ). Cold water immersion time was similar on all the cold stress trials $(32 \pm 17 \min , P=.55)$.

\section{COLD AIR EXPOSURE THERMAL RESPONSES}

At the start of the cold air exposure, core and skin temperature and metabolic heat production were similar $(P=.12, P=.38$, and $P=.69$, respectively $)$. Irrespective of the cold casualty treatment method employed, core temperature was similar during the 3hour cold air exposures $(P=.33)$. Core temperature declined to a low of $35.4 \pm 0.3^{\circ} \mathrm{C}$ within the first 20 minutes and then returned to within normal control core temperatures by the third hour of cold air exposure $(P=.38)$.

Core temperature rewarming was achieved by different thermal and metabolic effects that suggest lower cold stress with MPS and MPS $+\mathrm{HP}$ than $\mathrm{P}$ and $\mathrm{P}+\mathrm{HD}$ (Figure 2, Table 1). Lower peripheral cold stress was clear from the 2.5 to $3.1^{\circ} \mathrm{C}$ higher skin temperature with MPS and MPS+HP than P and P+HD $(10 \%-13 \%, P<$ .001 , large effect sizes $d>2.7$; Figure 2A, Table 1). Throughout the cold air exposure skin temperature was higher on MPS and MPS $+\mathrm{HP}$ than $\mathrm{P}$ and $\mathrm{P}+\mathrm{HD}$ in the first $(P<.05)$, second $(P<.01)$, and third hours $(P<$ .01). Skin temperature was lower than CON throughout all cold air exposures trials $(P<.01)$.

Metabolic heat production was lower when cold casualties used MPS and MPS + HP than P and P+HD (15\%-38\%, $P<.05$, large effect sizes $d>.9$; Figure 2B, Table 1). Further, when participants used MPS and MPS + HP, metabolic heat production was not different to CON in the second and third hour of the cold air exposure $(P=.21$ and .80 , respectively; Figure $1 C)$. In contrast, metabolic heat production was greater than CON in the third hour of the cold air exposure when participants used $\mathrm{P}$ and $\mathrm{P}+\mathrm{HD}(P=.01$ and $<.001$, respectively). 

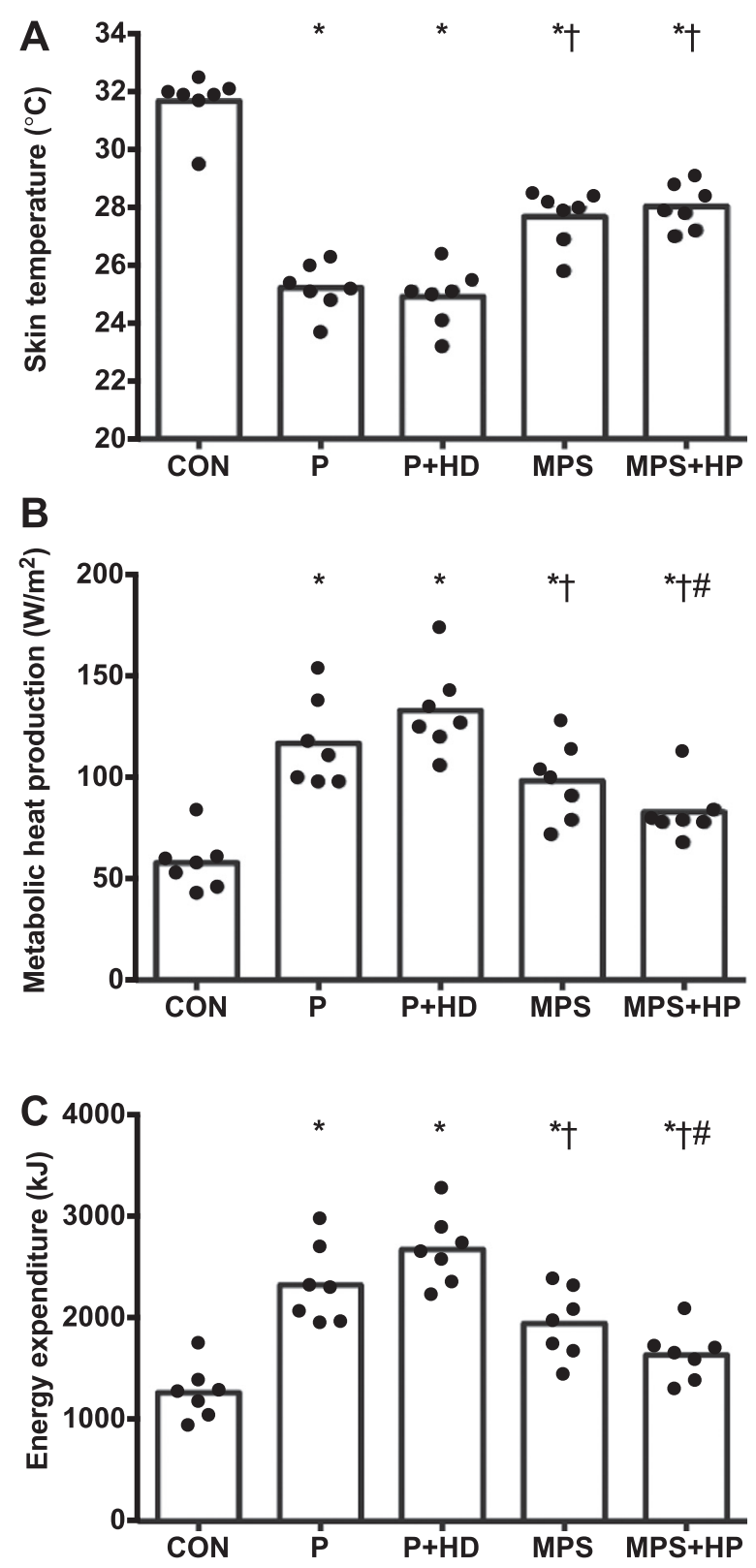

Figure 2. Mean thermal responses to a 3-hour cold air exposure $\left(0^{\circ} \mathrm{C}\right)$ when shivering cold casualties use a single-layered polyethylene survival bag $(\mathrm{P})$, a single-layered polyethylene survival bag with a hot drink $(\mathrm{P}+\mathrm{HD})$, a multi-layered MPS survival bag (MPS: Blizzard Survival bag), and a multi-layered MPS survival bag with 4 large chemical heat pads (MPS+HP: Blizzard Survival Heat bag). The fifth trial was a control $(\mathrm{CON})$ where participants remained seated in an ambient temperature $20.2 \pm 1.7^{\circ} \mathrm{C}$, relative humidity (RH) $41 \pm 6 \%$. Data are presented as individual responses (displayed as dot plots) and mean (as boxes). Statistical annotation are from post hoc multiple comparison tests to examine differences between treatment methods. * indicates difference to $\mathrm{CON}(P<.05)$. $\dagger$ indicates difference to $\mathrm{P}$ and $\mathrm{P}+\mathrm{HD}(\mathrm{P}<.05)$. \# indicates difference to MPS $(P<.05)$.
The addition of heat pads further lowered metabolic heat production by $15 \%$ (MPS+HP vs. MPS, $P=0.05$, large effect size $d=0.9$ ). Energy expenditure responses were similar to metabolic heat production (Figure 2C, Table 1). The addition of the hot drink to polyethylene survival bag did not increase skin temperature or lower metabolic heat production.

Subjective thermal discomfort was not statistically different between cold casualty treatments (thermal comfort $P=.36$, pain sensation to cold $P=.14$; Figure 3); however, MPS+HP was the only treatment where thermal discomfort was not greater than CON.

\section{Discussion}

This study compared the effectiveness of portable prehospital field methods to treat near-hypothermic $\left(35.4^{\circ} \mathrm{C}\right)$ shivering cold casualties in $0^{\circ} \mathrm{C}$ environment. Core temperature rewarming was accomplished $\left(\sim 1^{\circ} \mathrm{C}\right)$ by all methods within 3 hours. The prehospital methods, however, accomplished rewarming with a different peripheral cold stress and shivering thermogenesis. Near hypothermic cold casualties were rewarmed with less peripheral cold stress and shivering thermogenesis using a multi-layered, MPS survival bag compared with a polyethylene survival bag. Prehospital rewarming was further aided by large chemical heat pads. The differences in skin temperature and metabolic heat production observed between the prehospital methods should be considered meaningful and clinically relevant based on the observed large or very large effect size statistics. The differences between methods in the present study are also relatively large, 2- to 3-fold greater, when compared with another recent cold casualty study that evaluated the benefit of wet clothing removal or the addition of a vapor barrier. ${ }^{4}$

To the authors' knowledge, this is the first study to provide empirical evidence that a hot drink provides no rewarming benefit to a shivering cold casualty. As participants consumed approximately $1.3 \mathrm{~L}$ of $70^{\circ} \mathrm{C}$ flavored water, which likely represents the upper limits of what is practically portable, this study highlights that hot drinks have limited application for the treatment of cold casualties, and does not support prehospital accidental hypothermia guidelines to administer hot drinks to hypothermic patients. Instead guidelines should emphasize the consumption of sugary drinks irrespective of temperature. ${ }^{1}$

\section{LIMITATIONS}

In this study, participants were not blind to each prehospital method, which may have influenced their reports of thermal discomfort. Rectal core temperature 
Table 1. Differences in thermal responses and energy expenditure to a 3-h cold air exposure $\left(0^{\circ} \mathrm{C}\right)$ when shivering cold casualties use a single-layered polyethylene survival bag $(\mathrm{P})$, a single-layered polyethylene survival bag with a hot drink (P+HD), a multilayered MPS survival bag (MPS: Blizzard Survival bag), and a multi-layered MPS survival bag with 4 large chemical heat pads (MPS+HP: Blizzard Survival Heat bag)

\begin{tabular}{|c|c|c|c|c|}
\hline & $\begin{array}{c}\text { Mean absolute } \\
\text { difference }\end{array}$ & $\begin{array}{c}95 \% \text { confidence } \\
\text { interval of difference }\end{array}$ & Relative difference (\%) & Cohen's $d$ effect size \\
\hline \multicolumn{5}{|l|}{ Skin temperature $\left({ }^{\circ} \mathrm{C}\right)$} \\
\hline $\mathrm{P}+\mathrm{HD}$ vs. $\mathrm{P}$ & -0.3 & -0.7 to 1.3 & -1 & -0.3 (small) \\
\hline *MPS vs. P & 2.5 & 1.4 to 3.5 & 10 & 2.7 (large) \\
\hline${ }^{*} \mathrm{MPS}+\mathrm{HP}$ vs. P & 2.8 & 1.2 to 4.4 & 11 & 3.4 (large) \\
\hline${ }^{*}$ MPS vs. $\mathrm{P}+\mathrm{HD}$ & 2.8 & 1.6 to 3.9 & 11 & 2.8 (large) \\
\hline${ }^{*} \mathrm{MPS}+\mathrm{HP}$ vs. $\mathrm{P}+\mathrm{HD}$ & 3.1 & 1.8 to 4.4 & 13 & 3.4 (large) \\
\hline MPS+HP vs. MPS & 0.4 & -1.3 to 2.0 & 1 & 0.3 (small) \\
\hline \multicolumn{5}{|c|}{ Metabolic heat production $\left(\mathrm{W} / \mathrm{m}^{2}\right)$} \\
\hline $\mathrm{P}+\mathrm{HD}$ vs. $\mathrm{P}$ & 16 & -10 to 42 & 14 & 0.7 (medium) \\
\hline *MPS vs. P & -18 & -42 to 5 & -15 & -0.9 (large) \\
\hline${ }^{*} \mathrm{MPS}+\mathrm{HP}$ vs. P & -34 & -63 to -5 & -29 & -1.8 (large) \\
\hline${ }^{*}$ MPS vs. $\mathrm{P}+\mathrm{HD}$ & -35 & -48 to -21 & -26 & -1.7 (large) \\
\hline${ }^{*} \mathrm{MPS}+\mathrm{HP}$ vs. $\mathrm{P}+\mathrm{HD}$ & -50 & -69 to -31 & -38 & -2.7 (large) \\
\hline "MPS+HP vs. MPS & -15 & -37 to 6 & -15 & -0.9 (large) \\
\hline \multicolumn{5}{|l|}{ Energy expenditure $(\mathrm{kJ})$} \\
\hline${ }^{*} \mathrm{P}+\mathrm{HD}$ vs. $\mathrm{P}$ & 349 & -105 to 803 & 15 & 1.0 (large) \\
\hline *MPS vs. P & -380 & -871 to 110 & -16 & -1.0 (large) \\
\hline${ }^{*} \mathrm{MPS}+\mathrm{HP}$ vs. $\mathrm{P}$ & -34 & -1204 to -179 & -30 & -2.1 (large) \\
\hline${ }^{*}$ MPS vs. $\mathrm{P}+\mathrm{HD}$ & -35 & -1013 to -445 & -27 & -2.1 (large) \\
\hline${ }^{*} \mathrm{MPS}+\mathrm{HP}$ vs. $\mathrm{P}+\mathrm{HD}$ & -50 & -1379 to -684 & -39 & -3.4 (large) \\
\hline "MPS+HP vs. MPS & -15 & -729 to 108 & -16 & -1.0 (large) \\
\hline
\end{tabular}

* indicates statistical difference $(P<0.05)$ determined by uncorrected Fisher's LSD post hoc test. The $95 \%$ confidence interval of difference is from Tukey's post hoc test.

assessment has received recent criticism for monitoring cold casualties during rewarming because it is slower at responding than the esophageal method. ${ }^{1}$ The choice of a rectal thermistor in this study is less problematic because the interest was not to determine the precise temperature but to compare differences between prehospital methods. Further, skin temperature and metabolic heat production were the main outcome measures and not rewarming determined by core temperature. The performance of prehospital methods used in this study remains unclear in persons other than uninjured healthy young men (eg, women, children, elderly or injured persons). Encouragingly, it is likely that the differences between prehospital methods observed in uninjured healthy young men would be similar or even greater in persons other than uninjured healthy young men as they are at greater cold injury risk because of thermoregulatory disadvantages (ie, greater surface area, smaller total body mass and musculature, and impaired peripheral vasoconstriction and thermogenesis in older and injured persons). ${ }^{15}$

\section{PRACTICAL IMPLICATIONS}

The multi-layered MPS survival bag with heat pads should be the first choice to treat all casualties. Where it is not possible to carry the MPS survival bag with heat pads, the multi-layered MPS survival bag without heat pads should be chosen because of its superior performance compared with the single-layered, vapor-proof polyethylene survival bag.

\section{Conclusions}

In conclusion, near-hypothermic cold casualties are rewarmed with less peripheral cold stress and shivering thermogenesis using a multi-layered MPS survival bag compared with a polyethylene survival bag. Prehospital 


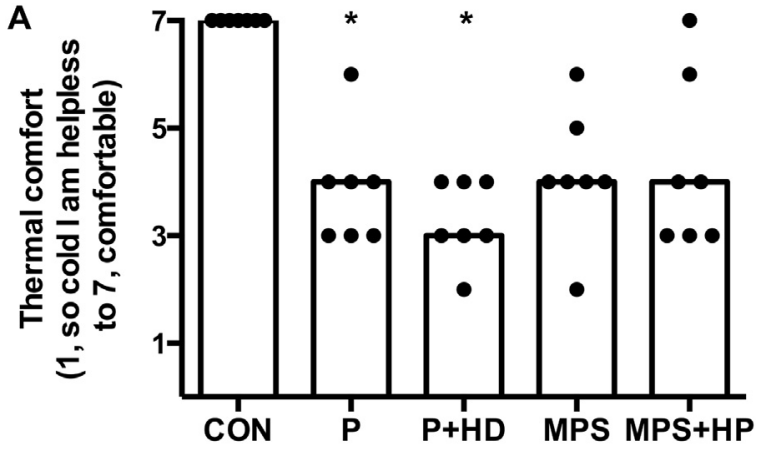

B

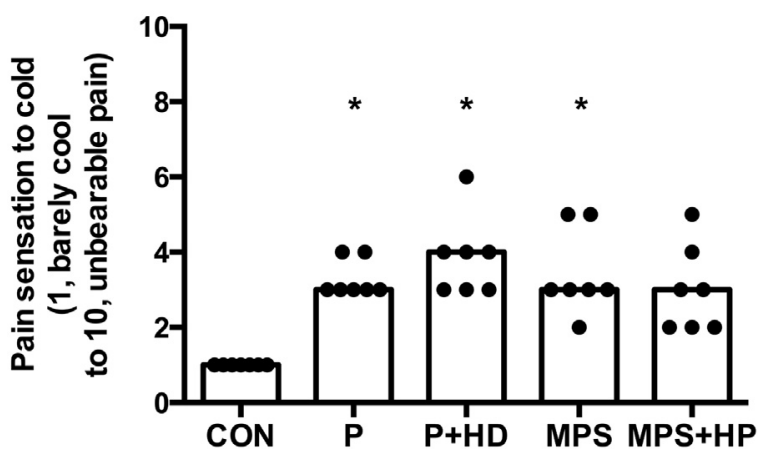

Figure 3. Mean thermal discomfort responses to a 3-hour cold air exposure $\left(0^{\circ} \mathrm{C}\right)$ when shivering cold casualties use a single-layered polyethylene survival bag $(\mathrm{P})$, a single-layered polyethylene survival bag with a hot drink $(\mathrm{P}+\mathrm{HD})$, a multi-layered MPS survival bag (MPS: Blizzard Survival bag), and a multi-layered MPS survival bag with 4 large chemical heat pads (MPS+HP: Blizzard Survival Heat bag). The fifth trial was a control $(\mathrm{CON})$ where participants remained seated in an ambient temperature $20.2 \pm 1.7^{\circ} \mathrm{C}$, relative humidity (RH) $41 \pm 6 \%$. Data are presented as individual responses (displayed as dot plots) and median (as boxes). Statistical annotations are from post hoc multiple comparison tests to examine differences between treatment methods. * indicates difference to $\mathrm{CON}(P<.05)$.

rewarming is further aided by large chemical heat pads but not by hot drinks.

\section{Acknowledgments}

The authors would like to thank the study participants for their efforts, Kevin Williams for his technical expertise, and Dr. Zoe Hoare for her statistical advice (Senior Statistician, North Wales Organisation for Randomised Trials in Health). The authors declare they have no financial relationships that may pose a conflict of interest.

JLB's PhD studies were supported by European Social Funding. Blizzard Protection Systems Ltd., Gwynedd, United Kingdom supplied all products gratis.

\section{References}

1. Zafren K, Giesbrecht GG, Danzl DF, et al. Wilderness Medical Society Practice Guidelines for the Out-ofHospital Evaluation and Treatment of Accidental Hypothermia. Wilderness Environ Med. 2014;25:S66-S85.

2. Langhelle A, Lockey D, Harris T, Davies G. Body temperature of trauma patients on admission to hospital: a comparison of anaesthetised and non-anaesthetised patients. Emerg Med J. 2012;29:239-242.

3. Thomassen $\varnothing$, Færevik H, Østerås $\varnothing$, et al. Comparison of three different prehospital wrapping methods for preventing hypothermia-a crossover study in humans. Scand $J$ Trauma Resusc Emerg Med. 2011;19:41.

4. Henriksson O, Lundgren P, Kuklane K, et al. Protection against cold in prehospital care: wet clothing removal or the addition of a vapor Barrier. Wilderness Env Med. 2015;26:11-20.

5. Giesbrecht G, Sessler D, Mekjavic I, Schroeder M, Bristow G. Treatment of mild immersion hypothermia by direct body-to-body contact. J Appl Physio. 1994;76: 2373-2379.

6. Lee J, Shirreffs S, Maughan R. Cold drink ingestion improves exercise endurance capacity in the heat. Med Sci Sport Exerc. 2008;40:1637-1644.

7. Lundgren JP, Henriksson O, Pretorius T, et al. Field torsowarming modalities: a comparative study using a human model. Prehosp Emerg Care. 2009;13:371-378.

8. ISO 9886, Ergonomics-Evaluation of Thermal Strain by Physiological Measurements. 2nd ed. Geneva, Switzerland: International Standards Organisation; 2004:1-21.

9. Gagge AP, Gonzalez R. Mechanisms of heat exchange: biophysics and physiology. In: Fregly MJ, Blatteis CM, eds. Handbook of Physiology, Section 4, Environmental Physiology. New York: Oxford University Press; 1996:45-85.

10. Weir JB. New methods for calculating metabolic rate with special reference to protein metabolism. J Physiol. 1949;109:1-9.

11. Oliver SJ, Harper Smith AD, Costa RJS, Maassen N, Bilzon JLJ, Walsh NP. Two nights of sleep deprivation with or without energy restriction does not impair the thermal response to cold. Eur J Appl Physiol. 2015;115: 2059-2068.

12. Hollies N, Goldman R. Clothing Comfort: Interaction of Thermal, Ventilation, Construction, and Assessment Factors. Science, Ann Arbor, MI; 1977:112.

13. Chen AC, Rappelsberger P, Filz O. Topology of EEG coherence changes may reflect differential neural network activation in cold and pain perception. Brian Topogr. 1998;11:125-132.

14. Cohen J. The t test for means. In: Statistical Power Analysis for the Behavioral Sciences. 2nd ed. Hillsdale, New Jersey: Lawrence Erlbaum Associates; 1988:19-26.

15. Castellani JW, Young AJ, Ducharme MB, Giesbrecht GG, Glickman EL, Sallis RE. American College of Sports Medicine position stand: prevention of cold injuries during exercise. Med Sci Sport Exerc. 2006;38:2012-2029. 